\title{
Bisdemethoxycurcumin Reduces Methicillin-Resistant Staphylococcus aureus Expression of Virulence-Related Exoproteins and Inhibits the Biofilm Formation
}

\author{
Shu Wang, Ok-Hwa Kang * and Dong-Yeul Kwon * (1) \\ Department of Oriental Pharmacy, College of Pharmacy and Wonkwang Oriental Medicines Research Institute, \\ Wonkwang University, Iksan 54538, Jeonbuk, Korea; wshu1996@gmail.com \\ * Correspondence: kangokhwa@daum.net (O.-H.K.); sssimi@wku.ac.kr (D.-Y.K.); Tel.: +82-63-850-6802 (O.-H.K.)
}

Citation: Wang, S.; Kang, O.-H.;

Kwon, D.-Y. Bisdemethoxycurcumin Reduces Methicillin-Resistant Staphylococcus aureus Expression of Virulence-Related Exoproteins and Inhibits the Biofilm Formation. Toxins 2021, 13, 804. https://doi.org/ $10.3390 /$ toxins 13110804

Received: 14 October 2021

Accepted: 13 November 2021

Published: 15 November 2021

Publisher's Note: MDPI stays neutral with regard to jurisdictional claims in published maps and institutional affiliations.

\begin{abstract}
Methicillin-resistant Staphylococcus aureus (MRSA) is a major pathogen of nosocomial infection, which is resistant to most antibiotics. Presently, anti-virulence therapy and anti-biofilm therapy are considered to be promising alternatives. In the current work, we investigated the influence of bisdemethoxycurcumin (BDMC) on the virulence-related exoproteins and the biofilm formation using a reference strain and clinic isolated strains. Western blotting, quantitative RT-PCR, and tumor necrosis factor (TNF) release assay were performed to assess the efficacy of BDMC in reducing the expression of Staphylococcus enterotoxin-related exoproteins (enterotoxin A, enterotoxin B) and $\alpha$-toxin in MRSA. The anti-biofilm activity of BDMC was evaluated through a biofilm inhibition assay. The study suggests that sub-inhibitory concentrations of BDMC significantly inhibited the expression of sea, $s e b$, and hla at the mRNA level in MRSA. Moreover, the expression of virulence-related exoproteins was significantly decreased by down-regulating accessory gene regulator $a g r$, and the inhibition of biofilms formation was demonstrated by BDMC at sub-inhibitory concentrations. Consequently, the study suggests that BDMC may be a potential natural antibacterial agent to release the pressure brought by antibiotic resistance.
\end{abstract}

Keywords: MRSA; $\alpha$-toxin; enterotoxin A; enterotoxin B; biofilm; bisdemethoxycurcumin

Key Contribution: In this work, we suggested for the first time that bisdemethoxycurcumin (BDMC), at sub-inhibitory concentrations, significantly decreased the expression of enterotoxin $\mathrm{A}$, enterotoxin $\mathrm{B}$, and $\alpha$-toxin by down-regulating related genes as well as inhibiting the biofilm formation in MRSA. BDMC might be a promising natural drug against methicillin-resistant Staphylococcus aureus (MRSA).

\section{Introduction}

Methicillin-resistant Staphylococcus aureus (MRSA) is a group of Gram-positive bacteria causing infections in communities and medical institutions worldwide, with a high prevalence and severe drug resistance [1,2]. MRSA causes skin and tissue infections to patients, which, in severe cases, can cause endocarditis, sepsis and necrotizing pneumonia [3]. Unfortunately, drug-resistant strains have been reported through the use of vancomycin and linezolid, which are the current guideline-advised treatments for MRSA [4]. Hence, it is necessary to continuously develop novel therapies, including the use of antimicrobial adjuvants, anti-virulence, and anti-biofilm antibacterial [5].

Staphylococcus aureus (S. aureus) produces a variety of toxins during the growing process, including hemolysins, enterotoxins, leukocidin and toxic shock syndrome toxin [6]. $\alpha$-Toxin ( $\alpha$-hemolysin) is an exocrine protein encoded by the hla gene, which is cytotoxic to a variety of host cells and is also a toxin that causes cell damage and death [7]. Moreover, several studies have confirmed $\alpha$-toxin production correlated significantly with MRSA biofilm formation [8]. Biofilm is a community of microorganisms attached to biotic and abiotic surfaces [9], exhibiting drug resistance to broad-spectrum antibiotics. This biofilm is 
recalcitrant in efforts to be eradicated by anti-bacterial agents, further limiting the efficacy of currently available antibiotics [10]. Biofilm formation is regulated by the quorumsensing (QS) system [11], and the hld gene is regarded as the most effective molecule in the Staphylococcal QS system [12]. The biofilm formation increases MRSA resistance to antibiotics by 10-10,000 times and is also the cause of many chronic MRSA infections [13]. Therefore, ideal antibacterial agents could inhibit biofilm formation.

Staphylococcal enterotoxins (SEs) are a highly toxic family of proteins, which could cause food poisoning and even toxic shock syndrome (TSS). The first enterotoxin SEA was isolated in 1959, and so far, 25 SEs (SEA-SElZ) have been described [14]. These peptides are classified as pyrogenic toxin superantigens (SAg), which can mobilize a large number of $\mathrm{T}$ cells [15], resulting in the release of a large number of pro-inflammatory cytokines (such as IL-6 and TNF- $\alpha$ ), leading to toxic shock syndrome (TSS) [16]. Characterized by high fever, rash and hypertension, it can rapidly progress to multiple organ failure and death, among which, Staphylococcal enterotoxin B (SEB) is a typical enterotoxin. It was developed as a biological weapon in the 20th century because of its high toxicity and stability [17].

Turmeric, which originated in Asia, has been used as a spice and traditional medicine for centuries [18]. The main biologically active component of turmeric is curcumin, including the structurally related analogs, desmethoxycurcumin, and bisdemethoxycurcumin (BDMC), of which BDMC is a polyphenolic curcuminoid, with a variety of pharmacological activities, such as anti-inflammatory, antioxidant and anti-cancer effects $[19,20]$. However, currently, the antibacterial activity of BDMC is rarely studied. In our previous study, the antimicrobial activity of BDMC against MRSA has been proved with a MIC value of as low as $7.8 \mu \mathrm{g} / \mathrm{mL}$, which was resistant to the reference strain ATCC 33591 and clinically isolated strains [21]. The antibacterial mechanism of MRSA was further studied in the current report. The inhibitory effect of sub-inhibitory concentrations of BDMC on the expression of $\alpha$-toxin and enterotoxin protein as well as the regulatory genes hla, agr A, sea and seb [22] have been studied. Thus, the inhibitory effect of BDMC on biofilm has been studied in the present work.

\section{Results}

2.1. BDMC Reduces the Expression of $\alpha$-Toxin and Inhibits the Expression of agr $A$ and hla in $S$. aureus

Western blot was examined to evaluate the level of expression of $\alpha$-toxin treated or untreated with BDMC of sub-inhibitory concentrations. After treatment for $4 \mathrm{~h}$, BDMC production of $\alpha$-toxin was determined to be significantly suppressed. In the presence of $1 / 8 \mathrm{MIC}$ of BDMC, the protein level of $\alpha$-toxin was only detected at $11 \%$. However, as the concentration of BMDC increases, protein levels no longer decrease significantly (Figure 1a). The transcriptional level of the $\alpha$-toxin gene hla and agrA was quantified by quantitative RT-PCR (qRT-PCR). The concentration of BDMC significantly downregulated the expression of $a g r A$ and hla as low as $0.9 \mu \mathrm{g} / \mathrm{mL}(1 / 8 \mathrm{MIC})$ (Figure $1 \mathrm{~b}, \mathrm{c}$ ).

\subsection{BDMC Inhibits Biofilm Formation and Downregulated the Expression of hld}

A crystal violet biofilm assay has been performed to evaluate the inhibition of BDMC in biofilm formation at sub-inhibitory concentrations (1/8 MIC, 1/4 MIC, and 1/2 MIC). Dosedependent BDMC inhibited biofilm formation significantly at sub-inhibitory concentrations. Specifically, at 1/2 MIC, BDMC inhibited biofilm formation of ATCC 33591, DPS-1, and DPS-2 by $78 \%, 82 \%$, and $98 \%$, respectively (Figure $2 \mathrm{a}$ ). The results indicated that the production of $\alpha$-toxin was positively correlated with the formation of MRSA biofilm. Additionally, the transcriptional level of the biofilm-related gene hld was quantified by qRT-PCR. BDMC at $3.9 \mu \mathrm{g} / \mathrm{mL}(1 / 2 \mathrm{MIC})$ significantly downregulated the expressions of hld (Figure 2b). 
(a)

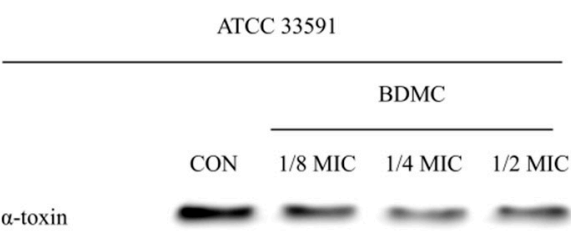

(b)

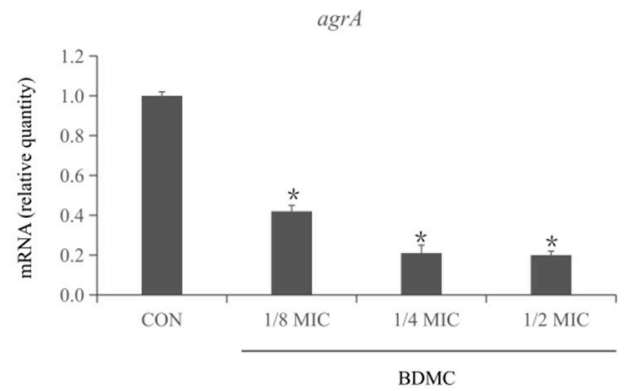

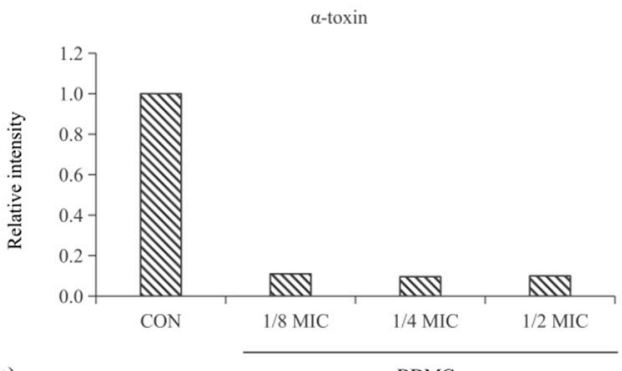

(c)

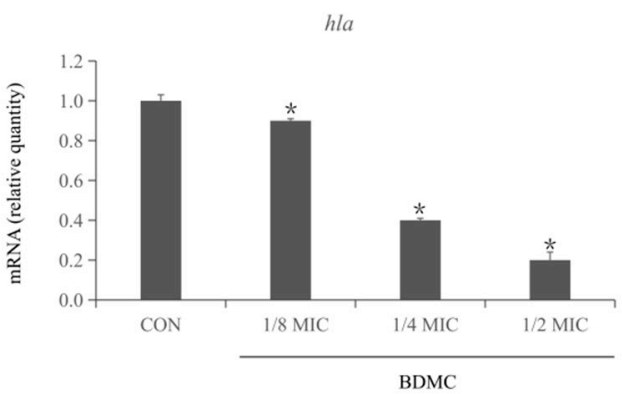

Figure 1. (a) Effect of BDMC treatment at sub-inhibitory concentrations on the expression of $\alpha$-toxin in MRSA (ATCC 33591). $\mathrm{CON}$ was a control treated without BDMC. After treatment for $4 \mathrm{~h}$, BDMC suppressed production of $\alpha$-toxin significantly was determined through Western blotting. $(\mathbf{b}, \mathbf{c})$ The inhibitory effect of BDMC on the expression of enterotoxin gene agr $A$ and hla was analyzed by qRT-PCR. After exposure to BDMC sub-inhibitory concentrations for $4 \mathrm{~h}$, the expression of agr $A$ and hla were down-regulated in a dose-dependent manner. The data were presented as the average of the three independent experiments with standard deviation. * Represents that $p<0.05$.

(a)

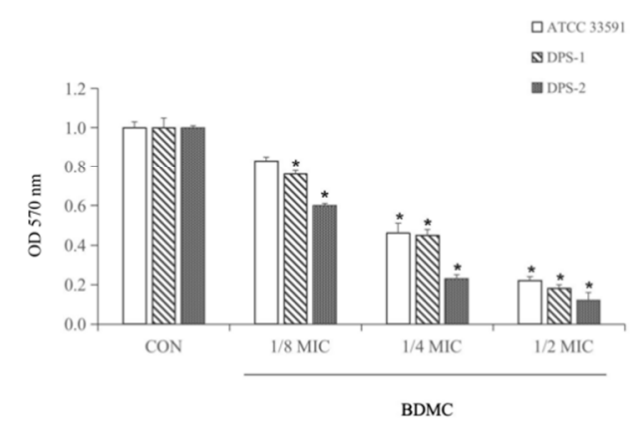

(b)

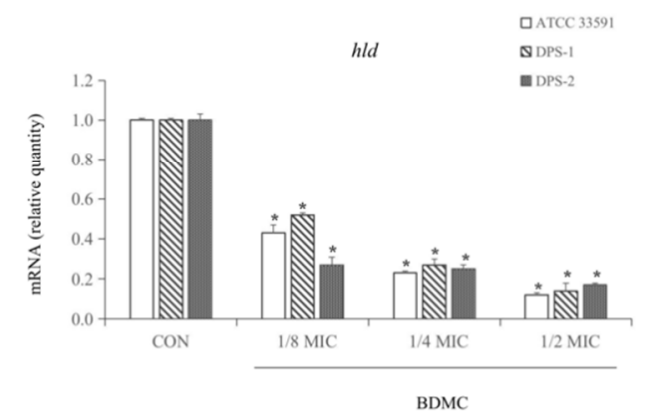

Figure 2. (a) Inhibitory effect of BDMC at sub-inhibitory concentrations on the biofilm formation of $S$. aureus standard strain (ATCC 33591) and clinical isolates DPS-1 and DPS-2. (b) In the presence of BDMC at sub-inhibitory concentrations, BDMC inhibited the expression of hld in MRSA (ATCC 33591, DPS-1, and DPS-2) cultures. The data were presented as the average of the three independent experiments with standard deviation. ${ }^{*}$ Represents that $p<0.05$.

\subsection{Expression of Staphylococcus Enterotoxin-Related Exoproteins in MRSA Treated with BDMC}

Western blot was performed to evaluate the level of expression of Staphylococcus enterotoxin-related exoproteins (enterotoxin A and enterotoxin B) treated or untreated with BDMC. After exposure to treatment for $4 \mathrm{~h}$, both exotoxins were inhibited by treating with sub-inhibitory concentrations of BDMC. The result showed that enterotoxin A and enterotoxin $\mathrm{B}$ were induced after being treated with 1/8 MIC of BDMC. In the presence of 1/4 MIC of BDMC, enterotoxin A was slightly inhibited, and enterotoxin B was significantly inhibited. Moreover, the production of enterotoxin B production in S. aureus ATCC 33591 was almost undetectable in the presence of $1 / 2 \mathrm{MIC}(3.9 \mu \mathrm{g} / \mathrm{mL})$ of BDMC (Figure 3a). 
(a)

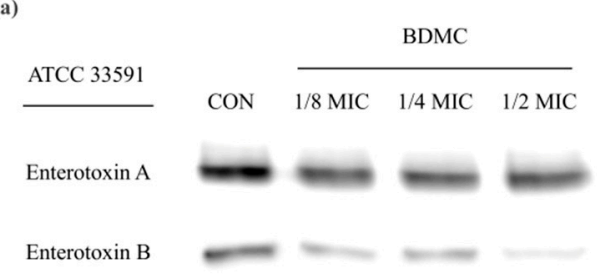

(b)

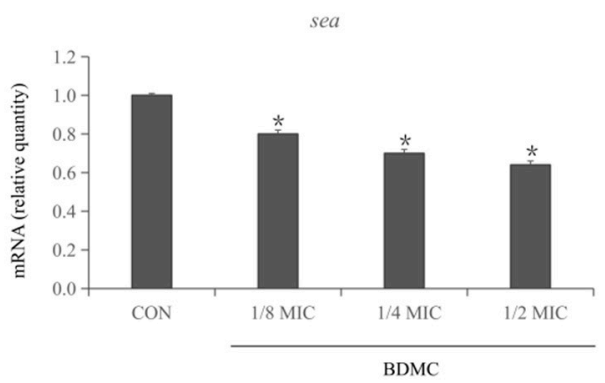

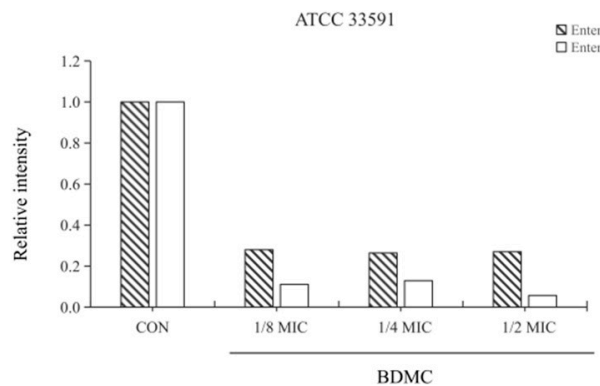

(c)

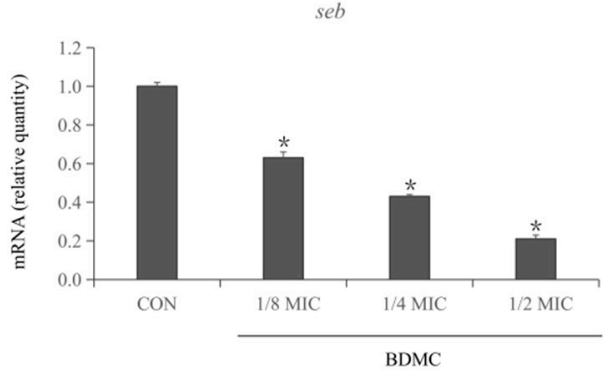

Figure 3. (a) BDMC at sub-inhibitory concentrations on enterotoxin A and enterotoxin B expression in MRSA (ATCC 33591). CON was a control treated without BDMC. (b,c) Relative expression of enterotoxin gene sea and seb in MRSA (ATCC 33591) cultures in the presence of sub-inhibitory concentrations of BDMC. Both enterotoxin genes were reduced in a dose-dependent manner. The data were presented as the average of the three independent experiments with standard deviation. * Represents that $p<0.05$.

\subsection{BDMC Downregulated the Expression of sea and seb in MRSA}

A qRT-PCR was performed to investigate the expression of the Staphylococcus enterotoxin genes (sea and seb) after treating with BDMC at sub-inhibitory concentrations. The expression of sea and seb was significantly down-regulated in S. aureus ATCC 33591 in a dose-dependent manner. The best inhibitory effect was shown after exposure to $1 / 2$ MIC $(3.9 \mu \mathrm{g} / \mathrm{mL})$ of BDMC. Meanwhile, the transcriptional level of sea and seb were decreased by 1.7 -fold and 5 -fold, respectively (Figure $3 b, c$ ).

\subsection{BDMC Reduces the Activity of IL-6 and TNF- $\alpha$}

As mentioned above, enterotoxin A and enterotoxin B, as superantigens, were produced by S. aureus, which could trigger an immune response, leading to proinflammatory cytokines. Enzyme-linked immunosorbent assay (ELISA) was used to determine the proinflammatory cytokines TNF- $\alpha$ and IL-6 levels in enterotoxin-stimulated RAW 264.7 cells, which grew in the culture supernatants of $S$. aureus that was treated with sub-inhibitory concentrations of BDMC. The result indicated that the expression of IL- 6 and TNF- $\alpha$ decreased in a dose-dependent manner. Moreover, the expression of TNF- $\alpha$ was significantly reduced (Figure $4 b$ ), and that the expression of IL-6 was slightly reduced (Figure 4a). 
(a)

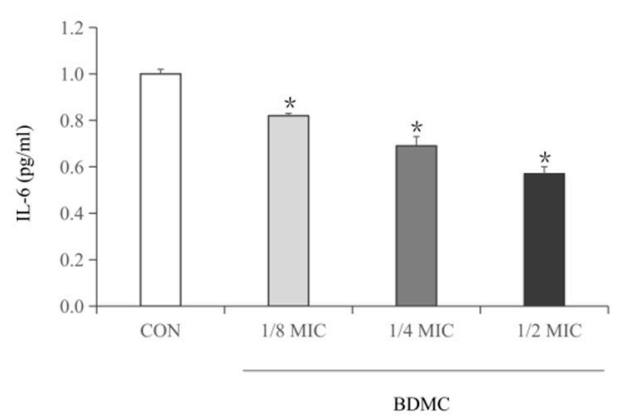

(b)

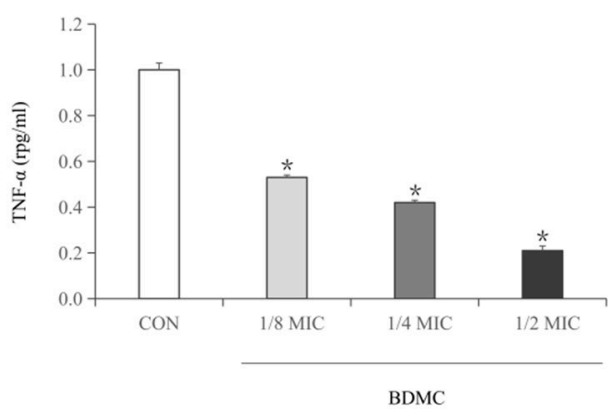

Figure 4. (a) IL-6 released from RAW 264.7 cells stimulated with S. aureus supernatants cultured in the presence of subinhibitory concentrations of BDMC. (b) TNF released from RAW 264.7 cells stimulated with S. aureus supernatants cultured in the presence of sub-inhibitory concentrations of BDMC. After stimulation for $24 \mathrm{~h}$ with RAW 264.7 cells, ELISA was used to measure the levels of IL- 6 and TNF. The data were presented as the average of the three independent experiments with standard deviation. * Represents that $p<0.05$.

\section{Discussion}

With the widespread appearance of virulent and multidrug-resistant MRSA strains, the morbidity and mortality caused by $S$. aureus infection had increased [23]. On the one hand, S. aureus produces a significant quantity of secreted toxins, which is suspected to be a potential mechanism for S. aureus to establish a chronic infection [24]. In the current study, we investigated the inhibitory effect of BDMC on $\alpha$-toxin, enterotoxine A and enterotoxin B of MRSA. The results indicated that sub-inhibitory concentrations of BDMC significantly inhibited the expression of hla at the mRNA level in MRSA and significantly reduced the $\alpha$-toxin production of $S$. aureus. Moreover, BDMC reduced the expression of Staphylococcal accessory gene regulator agrA. Therefore, we speculated that BDMC inhibits $\alpha$-toxin production by down-regulating the agr $A$ gene. The results of qRT-PCR showed that sub-inhibitory concentrations of BDMC down-regulated the expression of enterotoxin regulatory genes sea and seb, which was consistent with the results of inhibition of enterotoxin proteins indicated by Western blotting. The dose-dependent decrease in the level of pro-inflammatory cytokines TNF- $\alpha$ and IL-6 in RAW 264.7 cells grown in $S$. aureus treated with sub-inhibitory concentrations of BDMC, which strengthened the evidence of the inhibitory effect of BDMC on enterotoxin. According to the above results, we speculated that reducing the expression level of enterotoxin protein by down-regulating related genes was one of the reasons why BDMC could inhibit enterotoxin. In addition, it has been reported that enterotoxins could bind to polyphenols [25], and BDMC is a polyphenolic compound with two phenolic hydroxyl groups. The hydrophobicity and hydrogen bonding property of the phenolic hydroxyl group are believed to be easily combined with proteins and this chemical structure could be the second reason of the enterotoxins inhibition. Since drugs that resist virulence factors are considered not to affect viability, which may not destroy beneficial bacteria flora, inhibiting virulence factors of pathogens may be a promising strategy against MRSA [4].

On the other hand, since all currently available antibiotics will inevitably develop resistance, the development of novel antibiotic replacement therapies is urgently required. Naturally sourced antibacterial agents are considered to have extremely low drug resistance due to their rich sources and diverse and complex structures [26]. Based on this, we additionally conducted a preliminary trial of the BDMC resistance test, and the results showed that BDMC had no apparent resistance after 10 consecutive passages ( 45 days), and the resistance effect was better than that of the traditional antibiotic linezolid in the control group. Moreover, the US Food and Drug Administration (FDA) has approved curcuminoids as "Generally Recognized as Safe" (GRAS) [27], and it is assumed in the literature report dedicated to the evaluation of the biological activity of curcumin that BDMC is more stable 
than the other two derivatives (curcumin and dimethoxycurcumin) [28]. Additionally, it has also been proved in our previous studies that the anti-MRSA ability of BDMC is also more potent than that of the other two derivatives [21]. These advantages increase the possibility of BDMC as a substitute for antibiotics. However, the low solubility of curcumin in aqueous media, poor bioavailability, and pharmacokinetic defects limit its application in clinical treatment. Given the limitations of turmeric, approaches, such as lipid or nanoparticle materials combined with curcumin to make a mixture, have been reported [29].

Additionally, the formation of biofilms is one of the reasons why MRSA is difficult to eradicate [30]. Biofilms could enhance resistance to antibiotics, and biofilm-related infections are usually chronic or recurrent. Therefore, in the current requirements for antibacterial substances, not only should bacteria be inhibited for growth, but there should also be the ability to inhibit biofilm formation [31]. Studies have shown that, in addition to inhibiting toxins, plant-derived polyphenols can also inhibit the biofilm formation of food-related pathogens [32]. Based on the above, we hypothesized that BDMC could inhibit MRSA biofilm. The result was assumed that BDMC had a potent inhibitory effect on the biofilm formation of the clinical strain and the reference strain ATCC 33591 at sub-inhibitory concentrations, and the effective concentration was as low as $0.9 \mu \mathrm{g} / \mathrm{mL}$ (1/8 MIC). Moreover, BDMC significantly down-regulated biofilm formation-related gene hld, which emphasized the inhibitory effect of BDMC on biofilm formation. In addition, it was reported that the production of toxins is one of the reasons leading to the production of biofilm. Therefore, we speculated that BDMC might also inhibit $\alpha$-toxin production by the agr system, which would inhibit biofilm formation. Since biofilms are usually highly resistant to conventional antibiotics, the development of natural antibacterial substances with anti-biofilm activity may be of great significance [33].

In conclusion, our study first proposed the anti-toxin and anti-biofilm effects of BDMC, provided alternative or enhanced strategies for preventing and treating toxin-related infections, and provided a foundation for developing novel antibiofilm-specific antibiotics. Furthermore, our study revealed the potential of BDMC as a natural antibacterial agent, and a reference value was provided for the development of an antibiotic replacement, antibiotic adjuvant, or antibacterial lead compound. However, this study has certain limitations, such as the lack of a variety of tested strains and the lack of in vivo experiments. If the application of curcumin in clinical treatment is promoted, more in-depth in vivo experiments and the improvement of pharmacokinetics will be needed.

Furthermore, to solve the problem of MRSA infection, it is also critical to strengthen the solution to the occurrence of infection in communities. Reports have shown that the current spread of MRSA in the population is no longer caused by a large-scale nosocomial outbreak, and the proportion of community-related invasiveness as well as infections is higher [34].

\section{Materials and Methods}

\subsection{Reagents}

BDMC was purchased from Tokyo Chemical Industry Co.; Ltd. (Tokyo, Japan). Skim milk, Mueller-Hinton agar (MHA), and Mueller-Hinton broth (MHB) were obtained from Difco Laboratories (Baltimore, MD, USA). Anti-Staphylococcus enterotoxin A antibody, anti-Staphylococcus enterotoxin B antibody, and anti-Staphylococcus alpha-hemolysin antibody were acquired from Abcam (Cambridge, UK). Anti-rabbit IgG secondary antibody was acquired from Thermo Scientific Inc. (Waltham, MA, USA). Crystal violet was obtained from Sigma-Aldrich Co. (St. Louis, MO, USA). The sequences of primers used in qRT-PCR were listed in Table 1, which was acquired from Bioneer (Daejeon, Korea). 
Table 1. Primers used in in this study.

\begin{tabular}{|c|c|}
\hline Primer & Sequence $\left(5^{\prime}-3^{\prime}\right)$ \\
\hline $16 S$ & $\begin{array}{c}\text { F: ACTCCTACGGGAGGCAGCAG } \\
\text { R: ATTACCGCGGCTGCTGG }\end{array}$ \\
\hline sea & F: ATGGTGCTTATTATGGTTATC \\
\hline & R: CGTTTCCAAAGGTACTGTATT \\
\hline seb & F: TGTTCGGGTATTTGAAGATGG \\
\hline $\operatorname{agr} A$ & $\begin{array}{l}\text { F: TGATAATCCTTATGAGGTGCTT } \\
\text { R: CACTGTGACTCGTAACGAAAA }\end{array}$ \\
\hline hla & $\begin{array}{l}\text { F: TTGGTGCAAATGTTTC } \\
\text { R: TCACTTTCCAGCCTACT }\end{array}$ \\
\hline hld & $\begin{array}{l}\text { F: ATTTGTTCACTGTGTCGATAATCC } \\
\text { R: GGAGTGATTTCAATGGCACAAG }\end{array}$ \\
\hline
\end{tabular}

\subsection{Bacterial Strains and Growth Conditions}

In the report, one reference strain S. aureus ATCC 33591 (American Type Culture Collection, Manassas, VA, USA) was used as a reference strain and two clinical isolates of MRSA (DPS-1 and DPS-2) were isolated from patients at the Hospital of Wonkwang University was used. S. aureus was cultured in an incubator at $37^{\circ} \mathrm{C}$, and MHA or MHB was used as a solid medium or liquid medium, respectively.

\subsection{Western Blot Assay}

A Western blot assay was performed to evaluate the effect of BDMC on the expression of virulence-related exoproteins based on the previously reported investigation [21]. $S$. aureus strains (ATCC 33591) were cultured in MHB for $24 \mathrm{~h}$ and then treated for $4 \mathrm{~h}$ with sub-inhibitory concentrations of BDMC. Protein concentration was measured using a BioRad protein assay reagent (Bio-Rad Laboratories, Hercules, CA, USA) after centrifugation at $3000 \times g$ for $10 \mathrm{~min}$ to harvest cell protein extracts. The supernatant was separated by SDS-PAGE and electroblotted onto Amersham HybondTM-P membranes (GE Healthcare, Piscataway, NJ, USA) that had been blocked with 5\% skim milk and probed for $8 \mathrm{~h}$ at $4{ }^{\circ} \mathrm{C}$ with anti-Staphylococcus alpha-hemolysin antibody, anti-Staphylococcus enterotoxin A antibody, and anti-Staphylococcus enterotoxin B antibody (diluted 1:1000), and then reprobed by anti-rabbit IgG secondary antibody (diluted 1:1000) at room temperature for $2 \mathrm{~h}$. The membranes were then supplemented with $\mathrm{ECL}^{\mathrm{TM}}$ Prime Western Blotting Detection reagent (GE Healthcare Life Sciences, Incheon, Korea). The bands were visualized using the ImageQuant LAS-4000 mini chemical luminescent imager (GE Healthcare Life Sciences).

\subsection{Reverse Transcription and $q R T-P C R$}

A qRT-PCR was carried out according to the methods previously described [35]. Briefly, ATCC 33591 was cultured in MHB overnight and treated with sub-inhibitory concentrations of BDMC for $4 \mathrm{~h}$. The sample without BDMC was taken as a control. According to the manufacturer's protocol, total RNA was prepared from S. aureus (ATCC 33591) using the E.Z.N.A. ${ }^{\circledR}$ bacterial RNA kit (Omega Bio-tek, Norcross, GA, USA). The RNA concentration was evaluated by measuring the absorbance ratio at $260 \mathrm{~nm}$ on a spectrophotometer (BioTek, Winooski, VT, USA). Next, a QuantiTect reverse transcription kit (Qiagen, Dusseldorf, Germany) was used to synthesize the complementary DNA based on the manufacturer's instructions. PCR was set up to a total volume of $20 \mu \mathrm{L}$ as follows: $1 \mu \mathrm{L}$ of each primer $(10 \mu \mathrm{L} / \mathrm{mL}), 2 \mu \mathrm{L}$ of sample cDNA, $10 \mu \mathrm{L}$ of $2 \times$ SYBR Green PCR master mix (Life Technologies LTD, Warrington, UK), and $6 \mu \mathrm{L}$ of deionized water. StepOne software v2.3 produced from Applied Biosystems (Waltham, MA, USA) was used to calculate the expression level of the target gene relative to the endogenous reference gene 16 rRNA through the delta-delta cycle threshold method. 


\subsection{ELISA}

ELISA was performed using the previously described method [22]. Briefly, S. aureus ATCC 33591 in MHB was treated with sub-inhibitory concentrations of BDMC, and the untreated were taken as controls. The supernatants were collected after $4 \mathrm{~h}$ of incubation and $100 \mu \mathrm{L}$ was added into a 96 well culture plate with $100 \mu \mathrm{L}$ of RAW 264.7 cells. After incubating for $24 \mathrm{~h}$, the mixture was collected and analyzed based on the mouse TNF- $\alpha$ or IL-6 ELISA MAX standard set according to the instructions.

\subsection{Crystal Violet Biofilm Assay}

The inhibition of the BDMC on the biofilm formation of S. aureus DPS-1, DPS-2, and ATCC 33591 was established in a crystal violet biofilm assay using a previously described protocol [36]. To each well of 96-well microtiter plates, $100 \mu \mathrm{L}$ of overnight culture (0.5 MacFarland bacterial culture) was added and treated with sub-concentrations of BDMC. After $24 \mathrm{~h}$ at $37^{\circ} \mathrm{C}$, the planktonic cells were removed and washed with PBS three times, and each well of 96-well microtiter plates was stained with $1 \%(w / v)$ crystal violet for $10 \mathrm{~min}$ at room temperature, which was then rewashed for three times. The stained biofilms were solubilized in $100 \mu \mathrm{L}$ of absolute ethanol, and the optical density (OD) values at $570 \mathrm{~nm}$ were measured. The percentage of biofilm inhibition was estimated using the formula below.

Percentage of inhibition $=100-[($ OD $570 \mathrm{~nm}$ of the treatment wells $) /($ OD $570 \mathrm{~nm}$ of the control wells) $\times 100)]$.

\subsection{Statistical Analysis}

The analyses were carried out in triplicate, and data were given as the mean \pm standard deviation. The collected data were statistically analyzed using an independent Scheffe's $t$-test (SPSS software version 22.0; IBM SPSS, Armonk, NY, USA). A statistically significant $p$-value of less than 0.05 was evaluated.

Author Contributions: Conceptualization, D.-Y.K. and S.W.; methodology, D.-Y.K. and S.W.; software, O.-H.K.; validation, S.W., D.-Y.K. and O.-H.K.; formal analysis, S.W.; investigation, S.W.; resources, O.-H.K.; data curation, S.W.; writing—original draft preparation, S.W.; writing—review and editing, D.-Y.K. and O.-H.K.; visualization, S.W.; supervision, D.-Y.K. and O.-H.K.; project administration, D.-Y.K. and O.-H.K.; funding acquisition, D.-Y.K. All authors have read and agreed to the published version of the manuscript.

Funding: This research was funded by the National Research Foundation of Korea (NRF) grant funded by Korea government (MSIT) (no. 2020R1F1A1054227) and (NRF-2021R1I1A1A01058728). MSIT: Ministry of Science and ICT.

Institutional Review Board Statement: This research has been reviewed by the IACUC and it has been approved (Approved Number WKU18-34).

Informed Consent Statement: Patient consent was received and performed according to the guidelines of the Wonkwang University Institutional Review Board IRB.

Data Availability Statement: Personal information is included, so it was conducted for research only.

Conflicts of Interest: The authors declare no conflict of interest.

\section{References}

1. Yuan, Y.; Zai, Y.; Xi, X.; Ma, C.; Wang, L.; Zhou, M.; Shaw, C.; Chen, T. A novel membrane-disruptive antimicrobial peptide from frog skin secretion against cystic fibrosis isolates and evaluation of anti-MRSA effect using Galleria mellonella model. Biochim. Biophys. Acta (BBA)- Gen. Subj. 2019, 1863, 849-856. [CrossRef]

2. Dharmaratne, P.; Sapugahawatte, D.N.; Wang, B.; Chan, C.L.; Lau, K.M.; Lau, C.B.; Fung, K.P.; Ng, D.K.; Ip, M. Con-temporary approaches and future perspectives of antibacterial photodynamic therapy (aPDT) against methicillin resistant Staphylococcus aureus (MRSA): A systematic review. Eur. J. Med. Chem. 2020, 200, 112341. [CrossRef]

3. David, M.Z.; Daum, R.S. Community-Associated Methicillin-Resistant Staphylococcus aureus: Epidemiology and Clinical Consequences of an Emerging Epidemic. Clin. Microbiol. Rev. 2010, 23, 616-687. [CrossRef] 
4. $\quad$ El-Halfawy, O.M.; Czarny, T.L.; Flannagan, R.S.; Day, J.; Bozelli, J.C.; Kuiack, R.C.; Salim, A.; Eckert, P.; Epand, R.M.; McGavin, M.; et al. Discovery of an antivirulence compound that reverses $\beta$-lactam resistance in MRSA. Nat. Chem. Biol. 2020, 16, 143-149. [CrossRef]

5. Vestergaard, M.; Frees, D.; Ingmer, H. Antibiotic Resistance and the MRSA Problem. Microbiol. Spectr. 2019, 7, 2. [CrossRef]

6. Kathirvel, M.; Buchad, H.; Nair, M. Enhancement of the pathogenicity of Staphylococcus aureus strain Newman by a small noncoding RNA SprX1. Med. Microbiol. Immunol. 2016, 205, 563-574. [CrossRef]

7. Liu, L.; Shen, X.; Yu, J.; Cao, X.; Zhan, Q.; Guo, Y.; Yu, F. Subinhibitory Concentrations of Fusidic Acid May Reduce the Virulence of S. aureus by Down-Regulating sarA and saeRS to Reduce Biofilm Formation and $\alpha$-Toxin Expression. Front. Microbiol. 2020, 11, 25. [CrossRef]

8. Anderson, M.J.; Schaaf, E.; Breshears, L.M.; Wallis, H.W.; Johnson, J.R.; Tkaczyk, C.; Sellman, B.R.; Sun, J.; Peterson, M.L. Alpha-Toxin Contributes to Biofilm Formation among Staphylococcus aureus Wound Isolates. Toxins 2018, 10, 157. [CrossRef]

9. Ueda, Y.; Mashima, K.; Miyazaki, M.; Hara, S.; Takata, T.; Kamimura, H.; Takagi, S.; Jimi, S. Inhibitory effects of polysorbate 80 on MRSA biofilm formed on different substrates including dermal tissue. Sci. Rep. 2019, 9, 3128. [CrossRef]

10. Brady, R.A.; O'May, G.A.; Leid, J.G.; Prior, M.L.; Costerton, J.W.; Shirtliff, M.E. Resolution of Staphylococcus aureus Biofilm Infection Using Vaccination and Antibiotic Treatment. Infect. Immun. 2011, 79, 1797-1803. [CrossRef]

11. Naclerio, G.A.; Onyedibe, K.I.; Sintim, H.O. Lipoteichoic Acid Biosynthesis Inhibitors as Potent Inhibitors of S. aureus and E. faecalis Growth and Biofilm Formation. Molecules 2020, 25, 2277. [CrossRef]

12. Etter, D.; Schelin, J.; Schuppler, M.; Johler, S. Staphylococcal Enterotoxin C-An Update on SEC Variants, Their Structure and Properties, and Their Role in Foodborne Intoxications. Toxins 2020, 12, 584. [CrossRef]

13. Verreault, D.; Ennis, J.; Whaley, K.; Killeen, S.Z.; Karauzum, H.; Aman, M.J.; Holtsberg, R.; Doyle-Meyers, L.; Didier, P.J.; Zeitlin, L.; et al. Effective Treatment of Staphylococcal Enterotoxin B Aerosol Intoxication in Rhesus Macaques by Using Two Parenterally Administered High-Affinity Monoclonal Antibodies. Antimicrob. Agents Chemother. 2019, 63, e02049-18. [CrossRef]

14. Söderquist, B.; Källman, J.; Holmberg, H.; Vikerfors, T.; Kihlström, E. Secretion of IL-6, IL-8 and G-CSF by human endothelial cells in vitro in response to Staphylococcus aureus and staphylococcal exotoxins. APMIS 1998, 106, 1157-1164.

15. Chen, G.; Karauzum, H.; Long, H.; Carranza, D.; Holtsberg, F.W.; Howell, K.A.; Abaandou, L.; Zhang, B.; Jarvik, N.; Ye, W.; et al. Potent Neutralization of Staphylococcal Enterotoxin B In Vivo by Antibodies that Block Binding to the T-Cell Receptor. J. Mol. Biol. 2019, 431, 4354-4367. [CrossRef]

16. Wünsche, S.; Yuan, L.; Seidel-Morgenstern, A.; Lorenz, H. A Contribution to the Solid State Forms of Bis(demethoxy)curcumin: Co-Crystal Screening and Characterization. Molecules 2021, 26, 720. [CrossRef]

17. Duro-Castano, A.; Borrás, C.; Herranz-Pérez, V.; Blanco-Gandía, M.C.; Conejos-Sánchez, I.; Armiñán, A.; Mas-Bargues, C.; Inglés, M.; Miñarro, J.; Rodríguez-Arias, M.; et al. Targeting Alzheimer's disease with multimodal polypeptide-based nanoconjugates. Sci. Adv. 2021, 7, eabf9180. [CrossRef]

18. Jin, F.; Chen, X.; Yan, H.; Xu, Z.; Yang, B.; Luo, P.; He, Q. Bisdemethoxycurcumin attenuates cisplatin-induced renal injury through anti-apoptosis, anti-oxidant and anti-inflammatory. Eur. J. Pharmacol. 2020, 874, 173026. [CrossRef]

19. Wang, S.; Kim, M.-C.; Kang, O.-H.; Kwon, D.-Y. The Mechanism of Bisdemethoxycurcumin Enhances Conventional Antibiotics against Methicillin-Resistant Staphylococcus aureus. Int. J. Mol. Sci. 2020, 21, 7945. [CrossRef]

20. Mun, S.-H.; Kong, R.; Seo, Y.-S.; Zhou, T.; Kang, O.-H.; Shin, D.-W.; Kwon, D.-Y. Subinhibitory concentrations of punicalagin reduces expression of virulence-related exoproteins by Staphylococcus aureus. FEMS Microbiol. Lett. 2016, 363, 22. [CrossRef]

21. Miller, L.S.; Fowler, V.G.; Shukla, S.K.; Rose, W.E.; Proctor, R.A. Development of a vaccine against Staphylococcus aureus invasive infections: Evidence based on human immunity, genetics and bacterial evasion mechanisms. FEMS Microbiol. Rev. 2020, 44, 123-153. [CrossRef]

22. Fischer, A.J.; Kilgore, S.H.; Singh, S.B.; Allen, P.D.; Hansen, A.R.; Limoli, D.H.; Schlievert, P.M. High Prevalence of Staphylococcus aureus Enterotoxin Gene Cluster Superantigens in Cystic Fibrosis Clinical Isolates. Genes 2019, 10, 1036. [CrossRef]

23. Shimamura, Y.; Utsumi, M.; Hirai, C.; Nakano, S.; Ito, S.; Tsuji, A.; Ishii, T.; Hosoya, T.; Kan, T.; Ohashi, N.; et al. Binding of Catechins to Staphylococcal Enterotoxin A. Molecules 2018, 23, 1125. [CrossRef]

24. Rossiter, S.E.; Fletcher, M.H.; Wuest, W.M. Natural Products as Platforms to Overcome Antibiotic Resistance. Chem. Rev. 2017, 117, 12415-12474. [CrossRef]

25. Mashayekhi-Sardoo, H.; Mashayekhi-Sardoo, A.; Roufogalis, B.D.; Jamialahmadi, T.; Sahebkar, A. Impact of Curcumin on Microsomal Enzyme Activities: Drug Interaction and Chemopreventive Studies. Curr. Med. Chem. 2021, 28, 7122-7140. [CrossRef]

26. Miklášová, N.; Herich, P.; Dávila-Becerril, J.; Barroso-Flores, J.; Fischer-Fodor, E.; Valentová, J.; Leskovská, J.; Kožíšek, J.; Takáč, P.; Mojžiš, J. Evaluation of Antiproliferative Palladium(II) Complexes of Synthetic Bisdemethoxycurcumin towards In Vitro Cytotoxicity and Molecular Docking on DNA Sequence. Molecules 2021, 26, 4369. [CrossRef]

27. Kotha, R.R.; Luthria, D.L. Curcumin: Biological, Pharmaceutical, Nutraceutical, and Analytical Aspects. Molecules 2019, $24,2930$. [CrossRef]

28. Mihailescu, R.; Tafin, U.F.; Corvec, S.; Oliva, A.; Betrisey, B.; Borens, O.; Trampuz, A. High Activity of Fosfomycin and Rifampin against Methicillin-Resistant Staphylococcus aureus Biofilm In Vitro and in an Experimental Foreign-Body Infection Model. Antimicrob. Agents Chemother. 2014, 58, 2547-2553. [CrossRef] 
29. Shimamura, Y.; Hirai, C.; Sugiyama, Y.; Shibata, M.; Ozaki, J.; Murata, M.; Ohashi, N.; Masuda, S. Inhibitory effects of food additives derived from polyphenols on staphylococcal enterotoxin A production and biofilm formation by Staphylococcus aureus. Biosci. Biotechnol. Biochem. 2017, 81, 2346-2352. [CrossRef]

30. Qu, D.; Hou, Z.; Li, J.; Luo, L.; Su, S.; Ye, Z.; Bai, Y.; Zhang, X.; Chen, G.; Li, Z.; et al. A new coumarin compound DCH combats methicillin-resistant Staphylococcus aureus biofilm by targeting arginine repressor. Sci. Adv. 2020, 6, eaay9597. [CrossRef]

31. Takó, M.; Kerekes, E.B.; Zambrano, C.; Kotogán, A.; Papp, T.; Krisch, J.; Vágvölgyi, C. Plant Phenolics and Phenol-ic-Enriched Extracts as Antimicrobial Agents against Food-Contaminating Microorganisms. Antioxidants 2020, 9, 165. [CrossRef]

32. Coll, F.; Harrison, E.M.; Toleman, M.S.; Reuter, S.; Raven, K.E.; Blane, B.; Palmer, B.; Kappeler, A.R.M.; Brown, N.M.; Török, M.E.; et al. Longitudinal genomic surveillance of MRSA in the UK reveals transmission patterns in hospitals and the community. Sci. Transl. Med. 2017, 9, 413. [CrossRef]

33. Wang, S.; Luo, J.; Liu, X.; Kang, O.; Kwon, D. Antibacterial activity and synergy of antibiotics with sanguisorbigenin isolated from Sanguisorba officinalis L. against methicillin-resistant Staphylococcus aureus. Lett. Appl. Microbiol. 2021, 72, 238-244. [CrossRef]

34. Wang, S.; Kang, O.-H.; Kwon, D.-Y. Trans-Cinnamaldehyde Exhibits Synergy with Conventional Antibiotic against MethicillinResistant Staphylococcus aureus. Int. J. Mol. Sci. 2021, 22, 2752. [CrossRef]

35. Wang, J.; Jiao, H.; Meng, J.; Qiao, M.; Du, H.; He, M.; Ming, K.; Liu, J.; Wang, D.; Wu, Y. Baicalin Inhibits Biofilm Formation and the Quorum-Sensing System by Regulating the MsrA Drug Efflux Pump in Staphylococcus saprophyticus. Front. Microbiol. 2019, 10, 2800. [CrossRef]

36. Sharifi, A.; Mohammadzadeh, A.; Salehi, T.Z.; Mahmoodi, P.; Nourian, A. Cuminum cyminum L. Essential Oil: A Promising Antibacterial and Antivirulence Agent Against Multidrug-Resistant Staphylococcus aureus. Front. Microbiol. 2021, 12, 667833. [CrossRef] 